\title{
Thalidomide resurfaces for FDA consideration
}

Simply the mention of the drug thalidomide evokes for many searing images of deformed children with flippers instead of limbs, children born in 1959 and 1960 to women in the United Kingdom, Europe and Canada who had taken the drug during pregnancy as a sedative or to combat nausea. Although it has never been approved for any use in the United States, researchers there have been studying new and potentially significant therapeutic applications of the drug.

As a result of these new investigations, an advisory committee of the US Food and Drug Administration (FDA) met recently to discuss thalidomide's potential and its problems. The panel did not address whether the drug should be approved; its members only discussed its promise, and what should be done (if the drug is allowed on the market) to ensure that it is not taken by pregnant women.

AIDS specialists have found the drug useful against certain AIDS-related conditions, such as painful mouth ulcers (known as aphthous ulcers) and severe wasting. Thalidomide also has shown promise in treating glaucoma and lupus, as well as in graft-versus-host disease in cancer patients - a chronic rejection state that can be a deadly complication of bone marrow transplantation. And scientists think thalidomide eventually also could have an impact on a multitude of other ailments, including Crohn's disease, multiple sclerosis, arthritis, Kaposi's sarcoma, refractory psoriasis, and even Alzheimer's disease. The drug has also been used for years to treat leprosy in many regions where the disease is endemic, including Mexico, the Phillipines, South America and Asia.

"It is excruciatingly ironic that thalidomide - a drug that triggered a host of protections that govern drug research and regulation - should return," says Art Caplan, director of the Center for Bioethics at the University of Pennsylvania. Caplan believes that use of the drug will not be welcomed in those countries where it caused the most heartbreak, regardless of the passage of time. Many of those so-called "thalidomide babies" that are the drug's painful legacy are now adults, and hostile sentiments toward the drug are not likely to have ebbed. "I think it will be very tough to get that drug on the market in England, as compared to the US," Caplan predicts. "England was ground-zero of that disaster — we were not."

Thalidomide appears to work by suppressing the body's natural production of a substance called tumor necrosis factor (TNF), a potent cytokine that helps regulate the immune system and that fights bacterial infections. However, if TNF is active for too long or at too high a level, it can cause serious medical problems, including shock and body wasting.

Neither of the two small companies competing to bring thalidomide to market (Andrulis Pharmaceuticals in Beltsville, [MD], and Celigene in Warren, [NJ]) have submitted an application to the FDA, although this is expected soon. Peter Andrulis, chief of Andrulis Pharmaceuticals, describes the effects of thalidomide on aphthous ulcers in AIDS patients as "striking," and says he expects his company to seek approval for that indication in 1997.

Proponents of thalidomide believe it can escape its past as long as there are safeguards to prevent its teratogenic effects. Moreover, the FDA appears favorable to the idea of taking a second look, in the context of it new uses, as long as such protections are in place. " $\mathrm{A}$ 25-year-old patient with AIDS does not remember the tragedy of what happened in 1960," says FDA medical office Debra Birnkrant. But she adds that "if the data support that it's a promising therapy, we clearly could not deny approval just because of its history."

Thalidomide first became available overseas in 1958, and was touted as an extremely safe "miracle" drug because it induced a quick, calm sleep without hangover-like side effects. It also appeared to relieve nausea in pregnant women. But in 1959 and in 1960 in West Germany and England, thousands of infants began being born with gross deformities, most often shortened limbs that resembled flippers.

In 1961, before thalidomide was iden- tified as the cause of the birth defects seen in Europe, it first came before the FDA for approval. Frances Kelsey, a new FDA medical officer, was uneasy about the data and one of thalidomide's known side effects, nerve inflammation. She also was concerned about research indicating that the drug behaved differently in humans than in animals. Largely because of her fears, the drug was never approved in the United States. Furthermore, in the aftermath of the tragedy abroad, FDA's authority in regulating new drugs was further strengthened.

"It's definitely not a benign drug," Birnkrant says. However, she also notes that the diseases currently under study with thalidomide "are not benign illnesses." Birnkrant argues that for these diseases, "the risk/benefit equation changes. Thalidomide is not a drug for the general public."

Birnkrant and others stress that if the drug is eventually approved for some uses, the FDA would almost certainly require strongly worded labeling, as well as a widespread educational campaign to make women aware of its risks and to urge that, among other things, the drug never be shared with others. But education campaigns might not be enough. Despite an education campaign, a small number of pregnant women have used the anti-acne drug Accutane - another teratogen - and have given birth to babies with defects. Furthermore, in Brazil, where thalidomide is used in leprosy therapy, "there have been reports of thalidomide babies, and we're all concerned about that," Birnkrant says.

"We're going to be maniacal about controlling the distribution of the drug," Andrulis says. "We would seriously consider requiring pregnancy tests to get more drug. It's one thing to inform people; it's another thing to control the drug."

"It's obvious you have to educate women about its side effects because this drug is so dangerous to the fetus," he says. "I also think it's reasonable to ask women to abstain from sex, or use birth control, or even to have them tested each time they get a supply of the drug. That is the price one pays to use as nasty a drug as this."

Marlene Cimons Washington, $D C$ 